\title{
Combined Optical Connectivity and Optical Flow Velocimetry for interfacial characterisation of liquid atomisation
}

\author{
T. Wang* and Y. Hardalupas \\ Imperial College London, Mechanical Engineering Department, London SW7 2AZ, UK \\ ${ }^{*}$ Corresponding author email: tw5017@ic.ac.uk
}

\begin{abstract}
The Optical connectivity (OC) technique introduces a laser beam through an atomiser nozzle and relies on total internal reflection at the liquid interface to propagate inside the continuous liquid to study the instantaneous characteristics of the disintegrating continuous liquid and its interface during the primary atomisation through imaging the emitted fluorescent intensity from the liquid flow. In the current study, time-dependent $\mathrm{OC}$ is used to capture the temporal evolution of the liquid interface structures along the continuous liquid jet injected by a pressure jet atomiser into quiescent air at conditions leading to breakup within the first and second wind induced regime. Optical Flow Velocimetry (OFV) is developed to measure the local velocity of the interfacial structures of the liquid jet. The results show that the liquid interface accelerates downstream of the nozzle exit. The axial and radial mean and standard deviation of the fluctuations of the velocity of the interfacial structures are quantified. The results demonstrate the significance of liquid viscosity and liquid shear on atomisation.
\end{abstract}

\section{Keywords}

Optical connectivity, Optical flow velocimetry, Atomisation, Instability, Interfacial motion

\section{Introduction}

Atomisation disintegrates a continuous liquid fragment into fine droplets by external deformation due to shear and is important for many applications, including liquid fuel combustors, medical spraying or industrial coating. For example, fine fuel droplet sizes and spatially uniform droplet distributions are required in combustors to enhance combustion efficiency and reduce pollution through fast mixing of fuel vapour and oxygen.

The current study concentrates on pressure jet atomisers, which inject a liquid jet through an orifice with short length into quiescent air. Previous research in such atomisers shown that the liquid jet diameter, initial liquid flow velocity and liquid and gas properties, such as the viscosity and surface tension, affect the breakup process [1]. Moreover, the internal nozzle design and the liquid flow turbulence influence the atomisation process [2].

Ohnesorge [3] firstly proposed a regime map for the primary breakup of an injected liquid jet from pressure jet atomisers based on the Ohnesorge and Reynolds numbers (defined in the next section). Although the boundaries between the different regimes of [3] were modified due to experimental and theoretical studies [4-6], the qualitative behaviour of each regime remained the same. The boundaries divide the diagram into four regimes, namely Rayleigh, First Wind Induced, Second Wind Induced and Atomisation regimes respectively. The physical mechanisms determining the different regimes, including the initiation of interface instabilities and the contribution of liquid and gas turbulence on the interface structures of the liquid jet, are not yet understood despite their importance on atomisation.

Kelvin-Helmholtz linear instability, which is induced by the gaseous shear on the liquid jet interface [5-8], is broadly used to interpret the development of symmetric or asymmetric interfacial wave modes. Nevertheless, theoretical studies have limitations due to their assumptions and, for example, the asymmetric instability is associated with non-linear development. Therefore, experiments show discrepancies to the linear stability analysis. Meanwhile, experimental studies [9-13] reported that aerodynamic effects are not important for turbulent atomisation, but the thickness of the shear layer of the liquid flow at the nozzle exit dominates the process and determines the size of stripped-off droplets from the liquid interface [13]. This provides some evidence that the interfacial instability is not triggered by aerodynamic interaction and demonstrates the need for better understanding. 
Shinjo and Umemura [14] modelled a developing gaseous shear layer on the injecting liquid jet, and temporal tracking of the liquid surface pattern during jet injection suggested that the instability might be a Tollmien-Schlichting (TS) mode in the gaseous shear layer, which is similar to turbulent transition of solid-wall boundary layer. For case free of aerodynamic effect, Umemura [15] found that the deformation of the jet surface could be due to an instability of the thin liquid shear layer flow, which relaxes the liquid velocity profile produced by the nozzle wall.

The interfacial velocity at the surface of liquid jet is important for the understanding of the atomisation process, since it may indicate the velocity of the liquid instabilities on the surface and possibly of droplets immediately after breakup. However, no appropriate measuring technique is available to measure the interfacial velocity. For example, Particle Image Velocimetry (PIV) is not applicable, since it is not practical to seed the liquid flow with particle tracers and expect that they will be detected at the liquid surface in order to measure the interfacial velocity. Recently, the optical connectivity (OC) technique was developed to subside the optical limitations in detecting the continuous liquid core during primary breakup [16] and it was demonstrated to be more accurate in measuring the breakup length than shadowgraphic methods [17]. Meanwhile, by tracing the fluorescence intensity emitting from the 'light-seeded' liquid core, OC can be utilized to compute the interfacial velocity, which has been verified by Charalampous and Hardalupas [18], even though this study did not measure the instantaneous velocity distribution of the liquid interface structures. Subsequent studies based on OC provided measurements of instantaneous liquid breakup length, mean liquid interfacial velocity and liquid structure of a plain liquid jet and the spatial and temporal characteristics of the liquid interface structures in coaxial air-assisted atomisers $[18,19]$.

In this study, the optical connectivity (OC) is combined with optical flow velocimetry (OFV) to track the instantaneous liquid structures along the liquid interface in space and time and, for the first time, quantifies the spatial distribution of their instantaneous velocity and spatial acceleration, which demonstrates the importance of liquid viscosity on the atomisation process.

\section{Experiment set-up and methodology}

The nozzle of the current study is the same as in [18]. It has an orifice diameter $D=0.48 \mathrm{~mm}$ and length $L=2 \mathrm{~mm}$, leading to ratio $L / D$ of 4 . The short $L / D$ does not allow thick boundary layers to grow on the nozzle wall. In addition, no liquid cavitation occurs in the nozzle for the tested conditions, as confirmed from the pressure loss measurements and lack of cavitation bubbles within the liquid jet at the nozzle exit. More information can be found in [18].

The nozzle was mounted vertically to ensure a jet trajectory along a straight path, while the effect of gravity on liquid jet development can be neglected in the near field. The liquid jet was injected into quiescent air at room temperature and pressure. A pressure kettle (Spraying Systems) with deionised water was pressurised by compressed air constantly at 6 bar and its pressure was controlled by a pressure regulator with 0.3 mbar sensitivity. The pressure kettle delivered water flow to a rotameter through a stainless steel tubing and the rotameter metered the flowrate with uncertainty $\pm 3 \%$ by careful calibration.

Different water flowrates were studied, resulting in cross-sectional area averaged velocity $V_{L}$, between $3.6 \mathrm{~m} / \mathrm{s}$ and $21.6 \mathrm{~m} / \mathrm{s}$, adjusted in increments of $3.6 \mathrm{~m} / \mathrm{s}$. This increment of $V_{L}$ allowed the gradual observation of the liquid jet changes around the crossing from the first wind induced to the second wind induced atomisation regimes. Four non-dimensional parameters are considered, including gas Weber number $W e_{G}=\rho_{G} V_{L}^{2} D / \sigma$, liquid Weber number $W e_{L}=\rho_{L} V_{L}^{2} D / \sigma$, liquid Reynolds number $R e_{L}=\rho_{L} V_{L} D / \mu_{L}$ and Ohnesorge number $O h=\mu_{L} / \sqrt{\rho_{L} D \sigma}$. The air density $\rho_{G}$ and the liquid density $\rho_{L}$, viscosity $\mu_{L}$ and surface tension $\sigma$ are constant and the relevant non-dimensional parameters are listed in Table 1 for different $V_{L}$. Based on the Ohnesorge atomisation regime map [20], flow conditions 1-3 and 4-6 are in the first wind induced and second wind induced regimes respectively. 
Table 1. Experimental flow conditions and dimensionless numbers

\begin{tabular}{cccccc}
\hline Flow No. & $\boldsymbol{V}_{L}(\mathbf{m} / \mathbf{s})$ & $\boldsymbol{W e}_{G}$ & $\boldsymbol{W e}$ & $\boldsymbol{R e}_{L}$ & $\boldsymbol{O h}$ \\
\hline $\mathbf{1}$ & 3.6 & 0.1 & 85 & 1936 & $4.79 \times 10^{-3}$ \\
\hline $\mathbf{2}$ & 7.2 & 0.4 & 341 & 3871 & $4.79 \times 10^{-3}$ \\
\hline $\mathbf{3}$ & 10.8 & 1 & 767 & 5807 & $4.79 \times 10^{-3}$ \\
\hline $\mathbf{4}$ & 14.4 & 1.7 & 1363 & 7743 & $4.79 \times 10^{-3}$ \\
\hline $\mathbf{5}$ & 18 & 2.7 & 2130 & 9679 & $4.79 \times 10^{-3}$ \\
\hline $\mathbf{6}$ & 21.6 & 3.8 & 3067 & 11614 & $4.79 \times 10^{-3}$ \\
\hline
\end{tabular}

Figures $\mathbf{1}(\mathrm{a})$ and $\mathbf{1}(\mathrm{b})$ show plan and elevation views of the laser and optical arrangement of the optical connectivity (OC) technique [18]. Rhodamine WT dye was mixed with the deionised water in the pressure kettle with concentration of $1.61 \times 10^{-7} \mathrm{~mol} / \mathrm{L}$ in order to generate the optimum laser induced fluorescence for the $\mathrm{OC}$ technique. The concentration is according to proposition of Melton \& Lipp [21]. The laser beam from a New Wave double pulse Nd:YAG laser (120 mJ/pulse at $532 \mathrm{~nm}$ with initial diameter of $5 \mathrm{~mm}$ ) was reflected by mirrors to enter the nozzle through an optical window, mounted at the top of the nozzle. The laser light excited the Rhodamine WT molecules to emit fluorescence. The fluorescent intensities emitted from the liquid jet were imaged by a PCO Sensicam QE inter-frame camera (12 bit, $1376 \times 1040$ pixels). A Questar QM long distance telescope was mounted in front of the camera for high spatial resolution imaging. Since Rhodamine WT absorbs green light at $532 \mathrm{~nm}$ wavelegnth and emits fluorescence mostly at $587 \mathrm{~nm}$ wavelength, Schott OG 550 and OG 590 longpass optical filters, mounted behind the telescope, eliminated contributions from the laser and other undesirable sources. The spatial resolution of the captured images was $2.4 \mu \mathrm{m} /$ pixel with an observation area of $2.5 \mathrm{~mm} \times 3.3 \mathrm{~mm}$, which was appropriate for recording the full range of lengthscales of liquid jet interface instabilities.

The coordinate system is presented in Figure 1(c), the axial $Y$ and radial $X$ coordinates are along the streamwise and crosswise directions of the liquid jet. The observation range is from the nozzle exit, i.e. between $Y=0$ and $2.5 \mathrm{~mm}$ and radial range between $X=-1.65$ and $1.65 \mathrm{~mm}$. In this way, the recorded images quantify the motion and development of the liquid jet instability structures with axial distance from the nozzle exit.

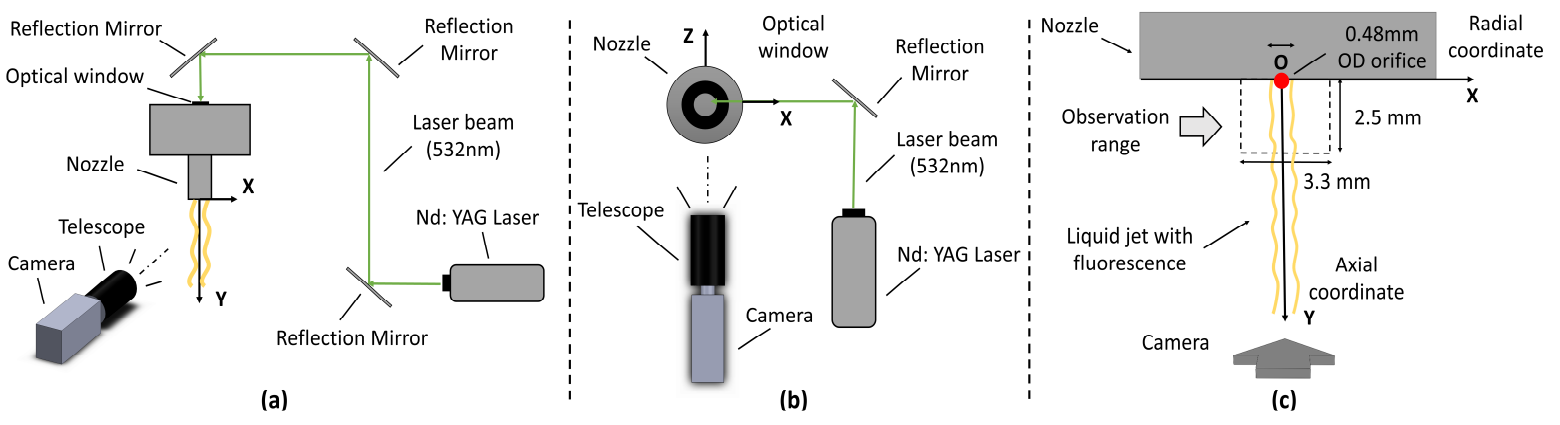

Figure 1. Schematic of experimental arragement. (a) Plan view. (b) Elevation view. (c) Coordinate system with its origin at the centre of the nozzle exit and the observation area of the liquid jet.

Using the optical connectivity (OC) technique, two time-delayed images of the fluorescent intensity emitted from the liquid jet are recorded, as shown in Figure 2(a). The double pulse PIV Nd:YAG laser, operating at $532 \mathrm{~nm}$ with $120 \mathrm{~mJ}$ per pulse was used with interval time between the pulses of $2 \mu \mathrm{s}$ to illuminate flows 1-3 at the first wind induced regime due to the lower liquid velocities, and $1 \mu$ s for the higher liquid velocities of flows 4-6 at the second wind induced regime. In Figure 2(a), the fluorescent intensity maxima in the interrogation window indicates a wavy or unsmooth structure on the liquid interface. Therefore, the crosscorrelation of the intensity distribution between subsequent images in time, similar to PIV algorithms, can quantify the displacement of the local intensity structures on the jet surface, which can then estimate the interfacial velocity. This approach, namely optical-flow velocimetry (OFV) or image correlation velocimetry (ICV), has been ultilized to track scalar 
fields with variable intensity, e.g. flame emission or stripped-off droplets from a liquid core [22-26]. The PIV software (Davis 8, LaVision) was used to compute the interfacial velocity by applying the cross-correlation to the fluorescent intensities of the $\mathrm{OC}$ images. A multi-passed cross-correlation algorithm was applied to minimize OFV measurement errors [22, 27]. A coarse interrogation window of $128 \times 128$ pixels with $50 \%$ window overlap was firstly applied to capture the movement of larger fluorescent intensity patterns. After capturing the movement of the larger scales, a second refined interrogation window of $32 \times 32$ pixels with $75 \%$ window overlap was used to capture the movement of smaller scales present within the first interrogation window. Therefore, the smallest resolved liquid structure was around 76.8 $\mu \mathrm{m}$, while 8 pixels (around $19.2 \mu \mathrm{m}$ ) exist between adjacent velocity vectors. Median filter and peak ratio were applied to reject spurious vectors.

(a)

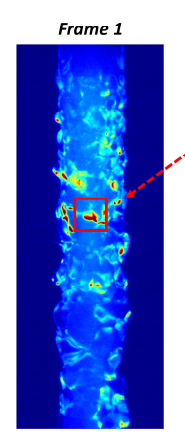

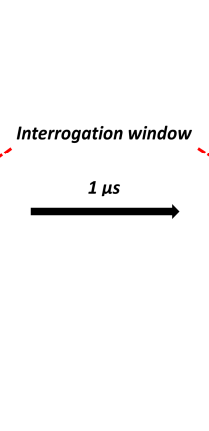

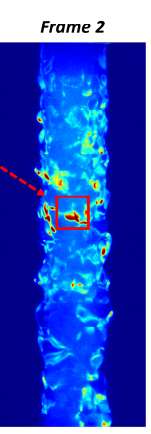

(b)

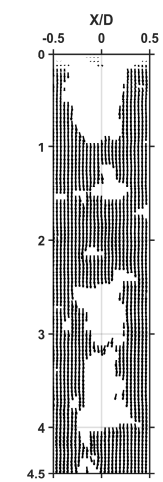

Figure 2. (a) Two $O C$ fluorescent intensity images of the liquid jet with $V_{L}=18 \mathrm{~m} / \mathrm{s}$ recorded with interval time 1 us. An example interrogation window is shown on the images, which allows the application of Optical Flow Velocimetry (OFV) algorithms to measure local velocity of the liquid interface structures. (b) Example instantaneous vector field computed by OFV for flow with $V_{L}=14.4 \mathrm{~m} / \mathrm{s}$.

The interfacial velocity was estimated using optical flow velocimetry (OFV) from 2000 instantaneous images for each flow condition. After exporting the vector maps from Davis 8, vectors with radial velocity component larger than the axial velocity component were identified as outliers, since the axial liquid velocity is expected to characterize the interfacial movement. Then, vectors with axial velocity component larger than $V_{L}$ or negative were also regarded as erroneous. In this way, spurious vectors are removed. At each OFV measurement point, the axial and radial components of the instantaneous velocity vectors are defined as instantaneous axial velocity $v(t)$ and radial velocity $u(t)$ respectively. $V$ and $U$ are the local mean axial and radial velocity computed from $v(t)$ and $u(t)$.

In order to demonstrate the performance of OFV, Figure 2(b) presents a measured instantaneous vector map with flow $V_{L}=14.4 \mathrm{~m} / \mathrm{s}$. The vector map shows that no vector can be detected at the region with smooth surface, since no variation of fluorescent intensity can be traced and thus the cross-correlation peak was not distinct and was rejected by the strict peak ratio. The smooth interface is mainly close to the nozzle exit and the middle line of the liquid jet with lower $V_{L}$, which was also observed in [18]. Therefore, the reliability of the combined OC and OFV technique (OC-OFV) for the quantification of the interfacial liquid movement was verified. The uncertainties of the local mean axial interfacial velocity are $4 \%$ for flows 1-3 in the first wind induced regime and 1\% for flows 4-6 in the second wind induced regime. The uncertainties for the local mean radial interfacial velocity are within \pm $0.12 \mathrm{~m} / \mathrm{s}$ and $\pm 0.07 \mathrm{~m} / \mathrm{s}$ for the flows $1-3$ and $4-6$ respectively. At the region where the jet surface is relative smooth, the maximum uncertainty can increase up to $8 \%$ for the local mean axial interfacial velocity and $\pm 0.5 \mathrm{~m} / \mathrm{s}$ for the mean radial interfacial velocity. These regions are close to the nozzle exit, and Figure 3 shows that the structures on the jet surface are very low in amplitude for $V_{L}=3.6 \mathrm{~m} / \mathrm{s}$ and $7.2 \mathrm{~m} / \mathrm{s}$, which increases the uncertainty. The statistical uncertainties are estimated from the number of validated vectors and standard deviation at each measurement point based on a $95 \%$ confidence interval. 


\section{Results and discussion}

The instantaneous OC fluorescent intensity images show that increased intensity is recorded at locations where evident deformations are formed at the liquid surface, which assists the tracking of the wavy structure of the liquid surface. This behaviour was demonstrated and explained by Charalampous \& Hardalupas [18] and it can be observed in Figure 3, which shows example OC images from different flow conditions. The emitted fluorescent intensity is relatively uniform close to the nozzle exit and becomes less uniform with the distance from the nozzle exit, while less uniform intensity appears closer to the nozzle for flows with increased $V_{L}$. For instance, the surface wrinkling is not dense and shows longer lengthscales between the variations of intensity for flows with $V_{L}=3.6$ and $7.2 \mathrm{~m} / \mathrm{s}$. With increasing $V_{L}$, fluorescent intensity variations become more common close to the nozzle exit and with a wider range of lengthscales along the entire liquid jet. It is also notable that interfacial waves with small wavelength start to emerge at the nozzle exit for increased $V_{L}$ (i.e. flows with $V_{L}=$ 18 and $21.6 \mathrm{~m} / \mathrm{s}$ ) and this agrees with Hoyt \& Taylor [11]. The wide range of wavelengths on the liquid surface demonstrates that the most amplified wavelength, estimated by theoretical instability analysis, cannot describe the physics of the atomisation process, while the physical process that determines the wide range of structure wavelengths is not clear.

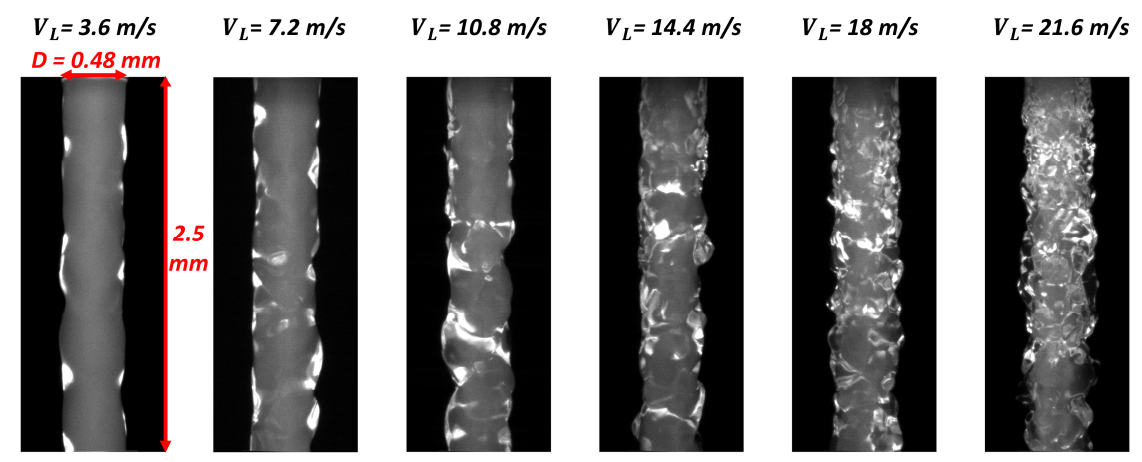

Figure 3. Example OC fluorescent intensity images of the liquid jet for different liquid velocities, obtained from the observation area shown in Figure 1.

The mean axial $(V)$ and radial $(U)$ interfacial velocity along the radial direction of the interface of the liquid jet can be measured with the OFV technique, as introduced in the previous section. The $V$ and $U$ radial profiles at $Y / D=2.5$ along the jet surface are presented in Figure 4(a). Since almost no intensity feature can be detected in the middle region of the jet surface for low $V_{L}$, profiles for flows with $V_{L}=3.6$ and $7.2 \mathrm{~m} / \mathrm{s}$ are not shown. It is noted that the presented velocities are along the circumference of the liquid jet rather than along a radial profile that crosses the jet centreline, as commonly reported for jet flows. From $X / D=-$ 0.45 to $X / D=0.45$ at the jet surface, the mean axial velocity $V$ increases with $V_{L}$, which is reasonable, and remains constant along the jet profile, proving that the axial interfacial motion of the liquid jet is radially uniform. Meanwhile, the mean radial interfacial velocity $U$ is almost zero for all flow conditions, confirming, as expected, that the interfacial waves do not exhibit a preferential rotational direction in their movement. Therefore, for example, there is no helical instability that makes the liquid interface to rotate around the liquid jet axis. This is expected for an injected axisymmetric flow without mean tangential component.

The standard deviation (RMS) of the axial and radial interfacial velocity, noted as $V_{R M S}$ and $u_{R M S}$, is also quantified to evaluate the fluctuations of the interfacial velocity. Figure $\mathbf{4}(\mathbf{b})$ presents $v_{R M S}$ and $u_{R M S}$ along the jet surface at $Y / D=2.5$ of the flow with $V_{L}=18 \mathrm{~m} / \mathrm{s}$, while $V_{R M S}$ and $U_{R M S}$ are also normalized by the corresponding local $V$ in Figure 4(c). $\varepsilon_{R M S}=$ $\beta / \sqrt{2(n-1)}$ is used to estimate the uncertainty of RMS, where $\beta$ is the local RMS value. The maximum uncertainty of the RMS values is $\pm 4 \%$ based on a $95 \%$ confidence interval. Figure 4(b) shows that the radial profile of $u_{R M S}$ has maxima around the centre $(X / D=0)$ and decreases with radial distance, opposite to the relatively constant profile of $V_{R M S}$. The 
shape of the radial profile of $U_{R M S}$ can be explained by the 3-D jet surface geometry and the instantaneous motion of the interfacial structures. The radial interfacial velocity at the centre is calculated from the tangential motion of the interfacial structures, while the radial motion is detected close to the edge. Since the velocity $U$ should be zero along the jet surface, the radial interfacial velocity can be considered as the radial fluctuating velocity. Therefore, the radial velocity of the interfacial structure away from the jet centre will be lower than the values close to the jet centre, which results to a maximum value of $u_{R M S}$ at $X / D=0$ on the jet surface. The profile of $v_{R M S}$ remains constant along the jet interface, since the 3-D jet geometry and the observation direction do not affect the measurement of the axial interfacial velocity. Meanwhile, the values of $v_{R M S} / \mathrm{N}$ are between $0.07-0.08$ in Figure 4(c), indicating the magnitude of the velocity fluctuations of the mean axial interfacial velocity.

The local mean axial interface velocities $V$ are radially averaged across the jet radial circumference and denoted as $\langle V\rangle$. Figure 5(a) presents $\langle V\rangle$ for all flow conditions, showing the development of the interfacial motion with distance from the nozzle exit. The uncertainties of $\langle V\rangle$ are less than $\pm 1 \%$, except for liquid flows with $V_{L}=3.6$ and $7.2 \mathrm{~m} / \mathrm{s}$, which have a maximum uncertainty of $\pm 3.5 \%$. The first point of the interfacial velocity measurements is not at $Y / D=0$, which is exactly at the nozzle exit, and therefore does not initiate from a velocity of $0 \mathrm{~m} / \mathrm{s}$, as expected to be due to the non-slip condition on the nozzle wall. This happens because the recorded images cannot detect the point at the nozzle exit. Therefore, a zero velocity value has been added at $Y / D=0$ of Figure 5(a) to match the nonslip condition at the solid wall. Figure 5(a) shows that the interfacial velocity accelerated with distance from the nozzle exit and became nearly constant by the end of the observation region. A sharp non-linear increase occurs close to the nozzle (up to $Y / D=2$ ), followed by an approximate linear increase (from $Y / D=2$ to $Y / D=4$ ). After $Y / D=4$, the axial interfacial velocities become almost constant for the slower flow, ending the interfacial acceleration.

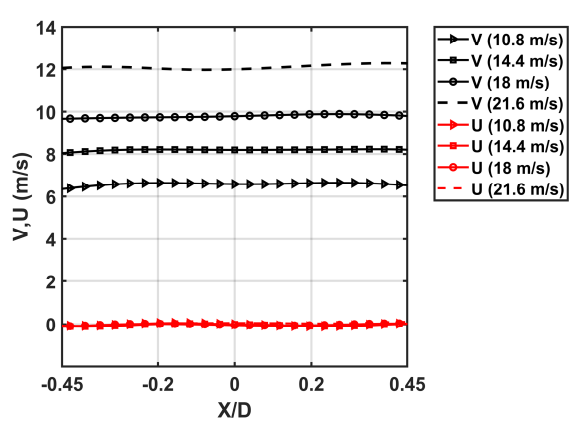

(a)

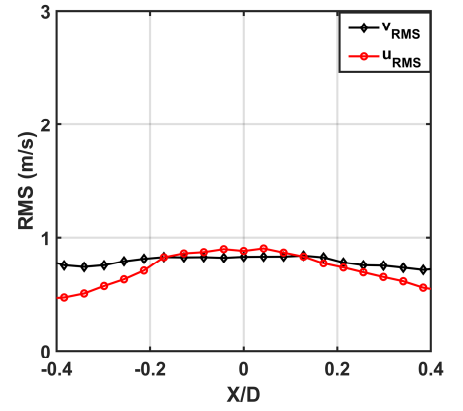

(b)

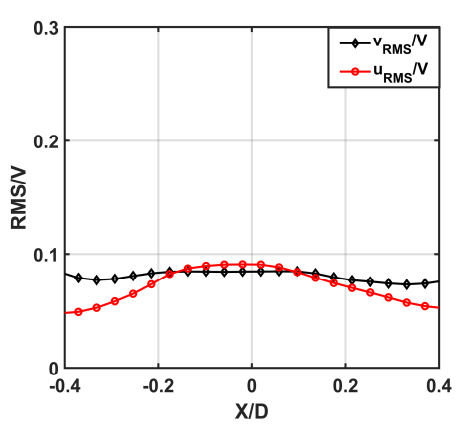

(c)

Figure 4. (a) Mean axial and radial interfacial velocity along the circumference at $Y / D=2.5$ of the liquid jet surface. (b) RMS values of interfacial velocity fluctutions of $v(t)$ and $u(t)$ along the circumference at $Y / D=2.5$ of the liquid jet surface for flow with $V_{L}=18 \mathrm{~m} / \mathrm{s}$. (c) Normalized $R M S$ values by the local mean axial velocity along the circumference at $Y / D=2.5$ of the liquid jet surface for flow with $V_{L}=18 \mathrm{~m} / \mathrm{s}$.

(a)

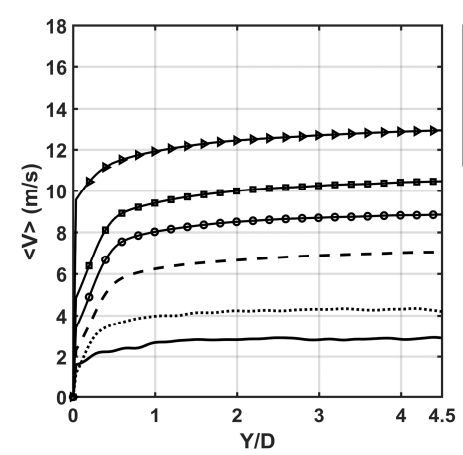

(b)

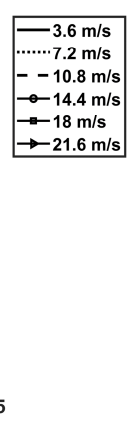

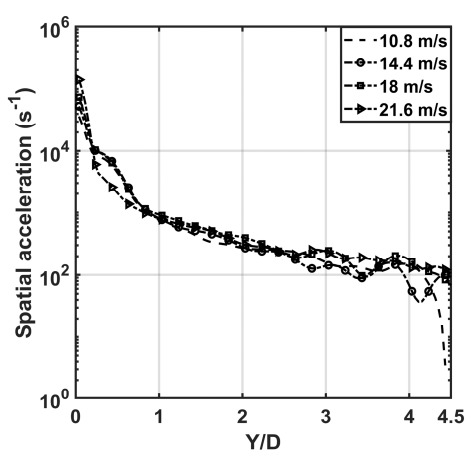

Figure 5. (a) Radially averaged axial interfacial velocity $\langle V\rangle$ as a function of axial distance from nozzle exit for all flow conditions of Table 1. (b) Spatial acceleration of the liquid jet interface as a function of axial distance from nozzle exit for flow condiions 3-6 of Table 1. 
The velocity deficit along the jet interface, observed in Figure 5(a), supports the influence of the annular boundary layer of the liquid jet, formed on the nozzle wall before being injected in the stationary gas, on the development of the shear layer below the liquid interface downstream of the nozzle exit. This spatial evolution of the liquid velocity can be responsible for the initial perturbation of the liquid interface, which was proposed by Umemura [15]. Therefore, the steep increase of $\langle V\rangle$ close to the nozzle exit is the result of the initial sharp velocity gradient of the shear layer in the liquid jet. The entire acceleration process, meanwhile, implies a relaxation of liquid shear layer and demonstrates the competition between internal liquid viscous force and external gaseous shear across the interface, as suggested by $[15,18]$. The interface velocity $\langle V\rangle$ is always lower than the area-averaged liquid velocity $V_{L}$, as expected. However, the approximately uniform value of the interfacial velocity beyond an axial distance of around $2 D$ is closer to the liquid velocity $V_{L}$ for low velocities than for high velocities (i.e. the ratio of $\langle V\rangle / V_{L}$ for $V_{L}=3.6 \mathrm{~m} / \mathrm{s}$ is around $83 \%$ and becomes around $61 \%$ for $V_{L}=21.6 \mathrm{~m} / \mathrm{s}$ ). It can be explained by the larger gaseous shear imposed on the liquid jet surface for higher $\langle V\rangle$. Since the liquid jet injected with higher $V_{L}$ always has larger magnitude of interfacial velocity $\langle V\rangle$, the stronger gaseous shear induced by the higher $\langle V\rangle$ may suppress the interfacial acceleration by the liquid viscous shear and therefore leads to a smaller ratio of $\langle V\rangle N_{L}$. The current ratio of $\langle V\rangle V_{L}$ is smaller than the results from Charalampous \& Hardalupas [18] for roughly the same $V_{L}$. This emphasizes the significance of the dynamic viscosity $\mu_{L}$ to the final state of the interfacial velocity, since the dynamic viscosity of the deionized water of the current study is lower than that of the hydrocarbon fuels studied in [15]. Hence, the more viscous liquid generates stronger internal viscous forces through the liquid shear layer and provides a more durable interfacial acceleration until the external gaseous flow shear balances the process.

The spatial acceleration can be calculated from the change of $\langle V\rangle$ over the distance between the OFV measurement points. The spatial acceleration results are plotted in Figure 5(b) against the distance from the nozzle exit for flows 3-6 of Table 1. The flows with $V_{L}=$ 3.6 and $7.2 \mathrm{~m} / \mathrm{s}$ are not included due to higher measurement uncertainty from the small changes of the interfacial velocity in space. The sudden decrease of the acceleration for $V_{L}$ $=10.8 \mathrm{~m} / \mathrm{s}$ at $Y / D$ around 4 in Figure 5(b) demonstrates that the acceleration is reduced to a small value as expected at this distance where the interfacial velocity remains constant. From Figure 5(b), the spatial acceleration for all flow conditions is maximum close to the nozzle exit and decays rapidly until $Y / D=2$, while the values remain relatively stable between $Y / D=2$ and $Y / D=4$ and may become very small after $Y / D=4$ (see the curve for flow with $V_{L}=10.8 \mathrm{~m} / \mathrm{s}$ ). These results agree with the observations for the interfacial velocity of Figure 5(a). Figure 5(b) also shows that the spatial acceleration for flows with different $V_{L}$ remains roughly the same, except after $Y / D=2.5$, where the acceleration is significantly smaller and the flow with higher $V_{L}$ has slightly higher spatial acceleration. However, if the corresponding timescales are considered, the flow with higher $V_{L}$ would have higher temporal acceleration, since the corresponding interface moves faster and spends short time within a considered spatial distance. This is also consistent with the characteristics of the shear layer, for which the faster flow always generates a sharper velocity gradient on the nozzle wall with short $L / D$ and therefore generates higher liquid viscous shear at the interface. Meanwhile, the measured higher axial interfacial velocity and larger interfacial acceleration lead to stronger aerodynamic interaction and momentum exchange between the liquid jet and the surrounding gas, which explain the increased wrinkling of the liquid jet surface for higher $V_{L}$ and increased number of wrinkling patterns near the nozzle exit.

\section{Conclusions}

The paper presented the interfacial characteristics of a liquid jet injected into quiescent air with different flow velocity. The optical connectivity (OC) technique allowed the detailed structures on the liquid jet interface to be recorded by using the fluorescent intensity, which demonstrated a more wrinkled jet interface for increased liquid jet velocity. By combining OC and optical flow velocimetry (OFV), the instantaneous, local interfacial velocities of the injected liquid jet were measured. The acceleration of the liquid interface velocity was 
quantified as a function of the axial distance from the nozzle exit, demonstrating the importance of the relaxation of the liquid shear layer due to liquid viscosity on the atomisation. The combined OC-OFV technique can be used to quantify the interfacial velocity in gas-liquid shear flows, which is not possible to obtain with other techniques.

\section{Acknowledgements}

The authors would like to thank Dr. G. Charalampous for the help with OFV. Discussions with Drs. C. Chen and Y. Liu are also acknowledged.

\section{References}

1. McCarthy, M. and N. Molloy, Review of stability of liquid jets and the influence of nozzle design. The Chemical Engineering Journal, 1974. 7(1): p. 1-20.

2. Birouk, M. and N. Lekic, Liquid jet breakup in quiescent atmosphere: A review. Atomization and Sprays, 2009. 19(6).

3. Ohnesorge, W.V., Die bildung von tropfen an düsen und die auflösung flüssiger strahlen. ZAMM-Journal of Applied Mathematics and Mechanics/Zeitschrift für Angewandte Mathematik und Mechanik, 1936. 16(6): p. 355-358.

4. Gordillo, J. and M. Pérez-Saborid, Aerodynamic effects in the break-up of liquid jets: on the first windinduced break-up regime. Journal of Fluid Mechanics, 2005. 541: p. 1.

5. Reitz, R. and F. Bracco, Mechanism of atomization of a liquid jet. The physics of Fluids, 1982. 25(10): p. 1730-1742.

6. Sterling, A.M. and C. Sleicher, The instability of capillary jets. Journal of Fluid Mechanics, 1975. 68(3): p. 477-495.

7. Rangel, R. and W. Sirignano, Nonlinear growth of Kelvin-Helmholtz instability: Effect of surface tension and density ratio. The Physics of fluids, 1988. 31(7): p. 1845-1855.

8. Weber, C., Zum zerfall eines flüssigkeitsstrahles. ZAMM-Journal of Applied Mathematics and Mechanics/Zeitschrift für Angewandte Mathematik und Mechanik, 1931. 11(2): p. 136-154.

9. Fenn III, R.W. and S. Middleman, Newtonian jet stability: the role of air resistance. AIChE Journal, 1969. 15(3): p. 379-383.

10. Hoyt, J. and J. Taylor, Turbulence structure in a water jet discharging in air. The Physics of Fluids, 1977. 20(10): p. S253-S257.

11. Hoyt, J.W. and J. Taylor, Waves on water jets. Journal of Fluid Mechanics, 1977. 83(1): p. 119-127.

12. Sallam, K., Z. Dai, and G. Faeth, Liquid breakup at the surface of turbulent round liquid jets in still gases. International Journal of Multiphase Flow, 2002. 28(3): p. 427-449.

13. Wu, P.K. and G. Faeth, Onset and end of drop formation along the surface of turbulent liquid jets in still gases. Physics of Fluids, 1995. 7(11): p. 2915-2917.

14. Shinjo, J. and A. Umemura, Surface instability and primary atomization characteristics of straight liquid jet sprays. International Journal of Multiphase Flow, 2011. 37(10): p. 1294-1304.

15. Umemura, A., Model for the initiation of atomization in a high-speed laminar liquid jet. Journal of fluid mechanics, 2014. 757: p. 665-700.

16. Charalampous, G., Y. Hardalupas, and A. Taylor, Structure of the continuous liquid jet core during coaxial air-blast atomisation. International Journal of Spray and Combustion Dynamics, 2009. 1(4): p. 389-415.

17. Charalampous, G., C. Hadjiyiannis, and Y. Hardalupas, Comparative measurement of the breakup length of liquid jets in airblast atomisers using optical connectivity, electrical connectivity and shadowgraphy. Measurement, 2016. 89: p. 288-299.

18. Charalampous, G. and Y. Hardalupas, How do liquid fuel physical properties affect liquid jet development in atomisers? Physics of Fluids, 2016. 28(10): p. 102106.

19. Charalampous, G., C. Hadjiyiannis, and Y. Hardalupas, Proper orthogonal decomposition of primary breakup and spray in co-axial airblast atomizers. Physics of Fluids, 2019. 31(4): p. 043304.

20. Reitz, R.D., Atomization and other breakup regimes of a liquid jet. PhDT, 1978.

21. Melton, L. and C. Lipp, Criteria for quantitative PLIF experiments using high-power lasers. Experiments in fluids, 2003. 35(4): p. 310-316.

22. Fielding, J., et al., Systematic errors in optical-flow velocimetry for turbulent flows and flames. Applied optics, 2001. 40(6): p. 757-764.

23. Komiyama, M., A. Miyafuji, and T. Takagi. Flamelet behavior in a turbulent diffusion flame measured by Rayleigh scattering image velocimetry. in Symposium (International) on Combustion. 1996. Elsevier.

24. Sedarsky, D., et al., Velocity measurements in the near field of a diesel fuel injector by ultrafast imagery. Experiments in fluids, 2013. 54(2): p. 1451.

25. Tokumaru, P. and P. Dimotakis, Image correlation velocimetry. Experiments in Fluids, 1995. 19(1): p. 115.

26. Sedarsky, D., et al., Fast-framing ballistic imaging of velocity in an aerated spray. Optics letters, 2009. 34(18): p. 2748-2750.

27. Adrian, L., R.J. Adrian, and J. Westerweel, Particle image velocimetry. 2011: Cambridge university press. 